\title{
Convergence and Divergence in the Neurochemical Regulation of Prepulse Inhibition of Startle and N40 Suppression in Rats
}

\author{
Neal R Swerdlow*,', Mark A Geyer', Jody M Shoemaker', Gregory A Light', David L Braff', \\ Karen E Stevens', Richard Sharp', Michelle Breier', Alaina Neary' and Pamela P Auerbach' \\ 'Department of Psychiatry, UCSD School of Medicine, La Jolla, CA, USA; ${ }^{2}$ Department of Psychiatry, University of Colorado Health Sciences \\ Center, Denver, CO, USA
}

\begin{abstract}
Prepulse inhibition of startle ('PPI'), a cross-species measure of sensorimotor gating, is impaired in schizophrenia patients. Suppression of P50 event-related potentials (ERPs) in response to the second of two clicks ('P50 gating') is also impaired in schizophrenia. Suppression of N40 ERPs to the second of two clicks ('N40 gating') is thought by some to be a rat homolog of human P50 gating. Emerging evidence suggests differences in the neurobiology of deficits detected by PPI vs P50 (or N40) gating. We recorded PPI and N40 gating contemporaneously in rats, to assess convergence and divergence in the neurochemical regulation of these measures. Dose-response studies examined the effects of apomorphine (APO), phencyclidine (PCP) or the $5 \mathrm{HT}_{2 \mathrm{~A}}$ agonist $\mathrm{DOI}$ on PPI, and on motor responses to stimuli (SI and S2) that elicit N40 gating. Effects of optimal drug doses on PPI and N40 gating were then assessed in other rats with implanted cortical surface electrodes. APO, PCP and DOI caused dose-dependent disruptions of both PPI and gating of motor responses to N40 stimuli. Reduced PPI reflected diminished prepulse effectiveness, demonstrated by increased startle levels on prepulse + pulse trials. In contrast, reduced gating of motor responses to $\mathrm{N} 40$ stimuli reflected a reduced motor response to SI. In separate rats, robust PPI, N40 potentials and N40 gating could be detected within one test. PPI and N40 gating were disrupted by APO, PCP, and DOI. Again, drug effects on PPI reflected increased startle on prepulse + pulse trials, while those on N40 gating reflected reduced ERP responses to $\mathrm{SI}$. In conslusion, when PPI and N40 gating were studied concurrently in rats, drug effects on PPI reflected reduced inhibition of startle by the prepulse, while diminished N40 gating reflected SI response suppression. Despite similarities in drug sensitivity, these results suggest that distinct neurobiological mechanisms underlie drug-induced deficits in PPI and N40 gating.

Neuropsychopharmacology (2006) 3 I, 506-5I5. doi:I0.1038/sj.npp. I30084I; published online I0 August 2005
\end{abstract}

Keywords: apomorphine; DOl; gating; N40 suppression; phencyclidine; prepulse inhibition; schizophrenia

\section{INTRODUCTION}

Deficits in the inhibition of behavioral and/or neural responses to sensory stimuli have been identified in schizophrenia patients using many experimental measures. Deficits in two of these inhibitory measures - prepulse inhibition of startle (PPI) and P50 event-related potential (ERP) suppression (P50 gating) - have been reported by several groups across the schizophrenia spectrum, including schizophrenia probands, their unaffected relatives, and schizotypal patients (Adler et al, 1982; Bolino et al, 1994; Boutros et al, 1991; Braff et al, 1978, 1999, 2001a; Cadenhead

*Correspondence: Dr NR Swerdlow, Department of Psychiatry, UCSD School of Medicine, 9500 Gilman Drive, La Jolla, CA 920930804, USA, Tel: + I 619543 6270, Fax: + I 619543 2493,

E-mail: nswerdlow@ucsd.edu

Received 4 April 2005; revised 10 June 2005; accepted 13 June 2005 Online publication: 21 June 2005 at http://www.acnp.org/citations/ Npp062 105050217/default.pdf et al, 2000a,b; Clementz et al, 1998; Erwin et al, 1998; Freedman et al, 1983, 1987, 1997; Judd et al, 1992; Kumari et al, 1999; Ludewig et al, 2002; Nagamoto et al, 1989; Oranje et al, 2002; Parwani et al, 2000; Siegel et al, 1984; Waldo et al, 1988; Weike et al, 2000; cf. Braff et al, 2001b; Braff and Freedman 2002). PPI is the reduction in startle magnitude that occurs when a weak prepulse precedes an intense, startling pulse by $30-300 \mathrm{~ms}$ (Graham, 1975). In humans, the eyeblink component of startle is most often assessed, using electromyographic recordings of the orbicularis oculi muscle. P50 suppression occurs when two brief identical clicks (commonly labelled 'S1' and 'S2') are presented with a $500 \mathrm{~ms}$ interstimulus interval. P50 waves are generated to each click, but there is normally a diminution of the second P50 wave relative to the first, attributable to the activation of inhibitory neural circuitry by the first P50 stimulus (Adler et al, 1982). P50 suppression is one of several ERP-related measures - together with N100 suppression and mismatch negativity (Boutros et al, 
2004; Light and Braff, 2005) — that are proving valuable for understanding information processing deficits in schizophrenia.

Historically, it has been assumed that reduced inhibition in PPI and P50 gating in schizophrenia reflect features of a common, unitary, underlying information-processing or 'gating' abnormality. Over the past decade, however, it has become clear that PPI and P50 gating have both overlapping (convergent) and separate (divergent) demographic, symptom, neurocognitive, and functional correlates, as well as separable and distinct neurobiological substrates, and probably different genetic architectures (Brenner et al, 2004; de Bruin et al, 2001a,b; Ellenbroek et al, 1999; Grunwald et al, 2003; Light and Braff 2001; Oranje et al, 1999; Schwarzkopf et al, 1993; Swerdlow et al, 2001a). Emerging from this work is the intriguing though speculative notion that groups of schizophrenia-spectrum patients may ultimately be distinguished on the basis of different patterns of convergent and divergent inhibitory deficits, and that these patterns may be associated with different neurobiological and genetic substrates and differing clinical profiles (cf. Braff and Freedman, 2002).

Both PPI and P50 ERP-suppression can be studied in animal models, as a way of understanding their neurobiological regulation. For example, pharmacological studies in rats reveals that PPI is reduced by direct and indirect DA agonists, NMDA antagonists, and direct and indirect 5HT agonists (Mansbach and Geyer, 1989; Sipes and Geyer, 1994; Swerdlow et al, 1986; cf. Geyer et al, 2001). Suppression of the N40 auditory ERP in rats ('N40 gating') is viewed by some to be homologous to P50 suppression in humans (Stevens et al, 1991, 1995, 1998), although there remains disagreement on this matter (Miyazato et al, 1999; Maxwell et al, 2004). Like PPI, N40 gating is reduced by direct and indirect DA agonists (Boutros et al, 1994; de Bruin et al, 2001a; Stevens et al, 1991, 1995). In a paradigm related to N40 suppression, hippocampal gating is disrupted by NMDA antagonists, including PCP (Miller et al, 1992; Shepard et al, 2003). There are, however, clear differences in the neurochemical regulation of PPI and N40 gating. For example, the disruptive effects of systemic DA agonists on N40 gating are reversed by D1 antagonists, apparently more potently than by D2 antagonists (Stevens et al, 1991). In contrast, the effects of DA agonists on PPI in rats are reversed preferentially by D2 blockers (Wan et al, 1996). Furthermore, the N40 gating-disruptive effects of the indirect $\mathrm{DA} / \mathrm{NE}$ agonist amphetamine are prevented by either $\alpha$-or $\beta$-noradrenergic blockade (Stevens et al, 1991), while its PPI-disruptive effects are not (Swerdlow et al, 2005). Unlike PPI, N40 gating is opposed by 5HT2 antagonists, instead of 5HT2 agonists (Johnson et al, 1998). Other findings utilizing ketamine (de Bruin et al, 1999), nicotine (Stevens et al, 1995) and nitric oxide synthetase (Adams and Stevens, 1998) underscore the different, and in some cases opposite neurochemical regulation of $\mathrm{N} 40$ gating and PPI.

Other strategies have been used to demonstrate divergence in these measures in rodents. For example, Ellenbroek et al (1999) reported a lack of significant correlations between PPI and ERP suppression in rats, when startle and ERPs were measured in separate sessions, 2 weeks apart. Their factorial analysis revealed four factors accounting for
$82 \%$ of the variance, with PPI and N40 ERP suppression each loading on separate factors.

Thus, while PPI and ERP-based gating measures may be of great importance to our understanding of schizophrenia, it remains unclear the degree to which these measures have overlapping vs separate neurobiological substrates and clinical implications. To provide more clarity in the areas of convergence and divergence of these measures, we assessed PPI and N40 gating in rats contemporaneously - that is, within the same test session-and tested the effects of the neurochemical manipulations of DA, NMDA and 5HT systems on these measures. Our findings demonstrate convergence in the sensitivity of these measures to drug manipulations, but also suggest divergence in the mechanistic basis for these apparently similar drug effects on PPI and N40 gating.

\section{MATERIALS AND METHODS}

\section{Experimental Animals}

Male Sprague-Dawley rats (225-250 g; Harlan, San Diego) were housed in a temperature-controlled room, in groups of 2-3 and maintained on a reversed 12:12 h light/dark schedule with ad lib food and water. Rats were handled individually within $48 \mathrm{~h}$ of arrival and again 1 week postsurgery. Surgery occurred between 7 and 10 days after arrival. All efforts were made to minimize animal suffering and reduce the number of animals used. All experiments conform to guidelines of the National Institute of Health for the use of animals in biomedical research (NIH Publications No. 80-23) and were approved by the Animal Subjects Committee at UCSD (protocol \#S01221).

\section{Surgical Preparations}

Rats received $0.1 \mathrm{ml}$ atropine sulfate subcutaneously (s.c.) (Vedco, $0.054 \mathrm{mg} / \mathrm{ml}$ ) 15-30 min before full anesthesia with sodium pentobarbital intraperitoneally (ip) (Abbott, $60 \mathrm{mg} /$ $\mathrm{kg}$ ) and placement in a Kopf stereotaxic instrument in a flat skull position (toothbar $3.3 \mathrm{~mm}$ below interaural line). A $2 \mathrm{~cm}$ incision was made to expose the skull; the periosteum was scraped until the bone surface was clean. Two holes were drilled for the ground wire placement on dura, approximately $1 \mathrm{~mm}$ anterior to Bregma and $1 \mathrm{~mm}$ on both sides of the midline suture. Three holes were drilled for anchor screws (Plastics One, Roanoke, VA, 0-80X3/32 in), and another hole was drilled for a Lomat electrode recording screw, placed $4 \mathrm{~mm}$ posterior to Bregma and $1 \mathrm{~mm}$ lateral to midline. Acrylic dental cement (A-M Systems, Carlsborg, WA, Dental Cement Powder, 525000 and Solvent, 526000) was used to firmly attach the ground wires, Lomat screw and anchor screws to the skull. Ground wires consisted of two 0.01 in thick Teflon-coated wires approximately 1 in in length that were crimped to a female amphenol pin. The Lomat recording electrode screw was soldered to a single 0.005 in thick Teflon-coated wire approximately 2 in in length and was also crimped to a female amphenol pin. Both pins were placed in the head plug that was then cemented to the skull. After the cement was completely dry, the incision was closed around the head plug. Rats were removed from the stereotaxic apparatus and 
allowed to fully recover on a heating pad before being placed in their home cages. The head plug was left available for attachment of the preamplifier cable for later EEG recordings.

\section{Behavioral Testing}

An initial set of studies was completed in unoperated rats $(n=17)$ to assess the behavioral sensitivity of rats to full dose-ranges of three drugs: (1) the direct DA agonist, apomorphine (APO); (2) the noncompetitive NMDA antagonist, phencyclidine (PCP); and (3) the $5 \mathrm{HT}_{2 \mathrm{~A}}$ agonist, (1-(2,5 dimethoxy-4-iodophenyl)-2-aminopropane (DOI)). While we have reported previously on these dose-response sensitivities with PPI, the present study differed significantly from past efforts due to the contemporaneous use of stimuli designed to elicit both PPI and N40 gating in the same test session. Thus, prior to actual measurement of N40 gating, PPI dose-sensitivities were verified in a test session that also included N40 'click pairs'. These studies also allowed us to assess the motoric effects of N40 click pairs, and the sensitivity of these responses to drug challenge. Based on these dose-response studies, a single active dose of each drug was selected for use in a test that involved contemporaneous measurement of PPI and N40 ERP gating.

For dose-response studies, startle chambers (SR-LAB, San Diego Instruments, San Diego, CA) were housed in a sound-attenuated room with a $60 \mathrm{~dB}(\mathrm{~A})$ ambient noise level; each consisted of a Plexiglas cylinder $8.2 \mathrm{~cm}$ in diameter resting on a $12.5 \times 25.5 \mathrm{~cm}^{2}$ Plexiglas frame within a ventilated enclosure. Noise bursts were presented via a speaker mounted $24 \mathrm{~cm}$ above the cylinder. A piezoelectric accelerometer mounted below the Plexiglas frame detected and transduced motion within the cylinder. The delivery of stimuli was controlled by the SR-LAB microcomputer and interface assembly that also digitized (0-4095), rectified, and recorded stabilimeter readings, with $1001-\mathrm{ms}$ readings collected at the beginning of stimulus onset. Startle amplitude was defined as the average of the 100 readings. Background noise and all acoustic stimuli were delivered through one Radio Shack Supertweeter (frequency response predominantly between 5 and $16 \mathrm{kHz}$ ) in each chamber. Other methodological details are in published materials (Geyer and Swerdlow, 1998).

For PPI/N40 ERP studies $(n=25)$, a single startle chamber was used (SR-LAB, San Diego Instruments, San Diego, CA) that included a modified Plexiglas cylinder with an elevated roof that allowed animals to move freely despite the presence of an EEG headpiece. The test chamber was also modified with electrical shielding, and an electrical interface cable that fastened to the EEG headpiece. Rats were acclimated to a similar test chamber for $30 \mathrm{~min} 1$ day prior to the matching session. Startle/ERP testing began 2 days later without drug injection, to demonstrate functionality of the ERP electrodes. Drug testing began 2-4 days later.

EEG signals were recorded via a preamplifier cable connection from the rat to an A-M Systems 2 Channel Microelectrode AC Amplifier (Carlsborg, WA, Model 1800). The recording cable contained three male pins at the proximal end, which connected to the preamplifier, and two male amphenol pins at the distal end of the cable, to connect with the female pins in the head plug. The filter settings on the amplifier were $0.1 \mathrm{~Hz}$ low cutoff and $50 \mathrm{~Hz}$ high cutoff. Notch filter was in 'Out' position; mode was set for 'record'; and gain was set at $10 \mathrm{~K}$. The amplified signals were recorded on the SR-LAB microcomputer.

To assign dose groups, rats were exposed to a brief 'matching' startle session 2-4 days prior to first testing, as reported previously (Geyer and Swerdlow, 1998). Rats were placed in a startle chamber, and exposed to $5 \mathrm{~min}$ of $70 \mathrm{~dB}$ background noise followed by 17 'PULSE' trials of $40 \mathrm{~ms}$ $120 \mathrm{~dB}$ noise bursts and three 'PREPULSE' trials consisting of a $20 \mathrm{~ms} 82 \mathrm{~dB}$ ( $12 \mathrm{~dB}$ over background) prepulse followed $100 \mathrm{~ms}$ by a $120 \mathrm{~dB}$ pulse (onset to onset). Average PPI levels were then used to assign rats to balanced dose groups.

For testing, rats were brought to the laboratory in their home cages, weighed, and placed in individual cages. Test sessions were approximately $35 \mathrm{~min}$ long and consisted of $5 \mathrm{~min}$ of $65 \mathrm{~dB}$ background followed by five startle trial types: PULSE noise bursts, PREPULSE trials $(20 \mathrm{~ms}$ noise bursts 70,75 , or $80 \mathrm{~dB}$ followed $100 \mathrm{~ms}$ by a PULSE) and NOSTIM trials (stabilimeter recordings obtained when no stimulus was presented). The session also included $60 \mathrm{~N} 40$ click pairs: $1 \mathrm{~ms} 85 \mathrm{~dB}(\mathrm{~A})$ white noise, separated by $500 \mathrm{~ms}$ with a fixed intertrial interval of $15 \mathrm{~s}$. The session consisted of initial (block 1) and final (block 6) blocks of three PULSE trials, separated by two blocks of 30 click pairs (blocks 2 and 5) and two blocks that included five PULSE trials and 15 PREPULSE trials (the latter divided equally among 70, 75, and $80 \mathrm{~dB}$ prepulse intensities) (blocks 3 and 4 ). 'NOSTIM' trials were interspersed between startle and PPI trials, which were used to assess gross motor activity during the test session, but were not included in the calculation of intertrial intervals, which were variable and averaged $15 \mathrm{~s}$. Reflex 'habituation' was determined based on the change in startle magnitude from the initial to the final block of PULSE trials.

EEG processing was performed offline using Neuroscan Reader Station v4.3.1 (Compumedics, El Paso, TX). Individual EEG responses to each click pair were high pass filtered ( $1 \mathrm{~Hz} ; 12 \mathrm{~dB} /$ octave rolloff), baseline corrected across the entire sweep, and manually screened for amplifier saturation or other artifacts. Waveform averaging was performed on artefact-free EEG epochs. The resultant averaged evoked potential waveforms were baseline corrected $(0-10 \mathrm{~ms})$. The $\mathrm{N} 40$ component, was calculated for averaged responses to the first (S1) and second (S2) clicks as the maximum negative waveform in the $20-60 \mathrm{~ms}$ range relative to the P20 component which was defined as the positive component that preceded the $\mathrm{N} 40$ or the maximum positive voltage in the $0-30 \mathrm{~ms}$ range. N40 gating was calculated as N40-P20 S2/N40-P20 S1.

\section{Drugs}

Dose-Response testing. APO (saline/ascorbate vehicle, 0.1, 0.25 , or $0.5 \mathrm{mg} / \mathrm{kg}$ s.c.) was administered to rats immediately prior to testing. PCP (saline vehicle, $0.5,1.0$, and $1.5 \mathrm{mg} / \mathrm{kg}$ ) and DOI (saline vehicle, $0.25,0.5$, or $1.0 \mathrm{mg} / \mathrm{kg}$ sc) were administered to rats $10 \mathrm{~min}$ prior to testing. For each drug, dose-response testing was completed using a within-subject design, with four tests each separated by 3-4 days, and drug order balanced. 
PPI/N40 testing. APO, PCP and DOI were administered as above, using only the vehicle and highest doses. All rats were first tested in a within-subject study of APO (vehicle $v s$ $0.5 \mathrm{mg} / \mathrm{kg}$ ), and were then tested two additional times, in between-subject studies of PCP (vehicle or $1.5 \mathrm{mg} / \mathrm{kg}$ ) and DOI (vehicle or $1.0 \mathrm{mg} / \mathrm{kg}$ ).

\section{Statistics}

Prepulse inhibition was defined as 100 -[(startle amplitude on prepulse + pulse trials/startle amplitude on PULSE trials) 100], and was analyzed by mixed-design analyses of variance (ANOVAs), with specific comparisons noted for each experiment. Separate analyses were performed using raw startle magnitude on PULSE and prepulse trials, to determine whether changes in percent PPI reflected a diminished ability of prepulses to inhibit startle. ANOVAs were also used to assess startle habituation (reduced startle magnitude between the initial and final blocks of PULSE trials) and cage displacement on NOSTIM trials. In doseresponse testing, chamber displacement was also recorded in the $100 \mathrm{~ms}$ epoch after each N40 click, and ANOVA was used to analyze click-induced motor responses to S1 and S2, as well as the S2/S1 ratio. Based on the low magnitude of these S1- and S2- associated chamber displacements $(<1 \%$ of startle magnitudes), additional analyses were performed after subtracting 'NOSTIM' baseline from the S1 and S2 motor signals. Measures of S1- and S2-associated cage displacements were not feasible during ERP acquisition, due to the ERP amplifier acquisition requirements. N40 amplitudes were analyzed by ANOVA, with trial $(\mathrm{S} 1, \mathrm{~S} 2)$ as a within-subject factor and dose as a within-subject factor for APO studies, and a between-subject factor for PCP and DOI studies. S2/S1 ratios were compared by one-way ANOVA; a small number of rats were excluded from ratio comparisons because $\mathrm{N} 40$ amplitude was reduced to zero under active drug conditions. Alpha was 0.05 for all statistical analyses.

\section{RESULTS}

\section{Study 1. Dose-Response Studies}

APO, PCP, and DOI produced dose-dependent disruptions of PPI (Figure 1). In each case, ANOVA revealed significant main effects of dose (F for APO, PCP, and DOI $=20.26$, 10.24, and 12.87, respectively; all $p<0.0001$ ) (Table 1a). Statistically significant reductions in PPI were evident with the $0.1,0.25$, and $0.5 \mathrm{mg} / \mathrm{kg}$ doses of APO, the 1.0 and $1.5 \mathrm{mg} / \mathrm{kg}$ doses of PCP, and the $0.25,0.5$, and $1.0 \mathrm{mg} / \mathrm{kg}$ doses of DOI.

Inspection of raw startle magnitude on PULSE and prepulse + pulse trials revealed that for APO and PCP, reduced PPI reflected a disruption in the ability of prepulses to inhibit startle, that is, a clear loss of sensorimotor gating (Swerdlow et al, 2000). Thus, startle magnitude on prepulse + pulse trials was increased significantly by these drugs, under conditions where startle magnitude on PULSE trials was not increased, and in some cases, was (nonsignificantly) reduced (Figure 2). These observations were supported statistically by ANOVAs, with no significant effects of dose on PULSE amplitude (F values for APO and PCP <1.2),
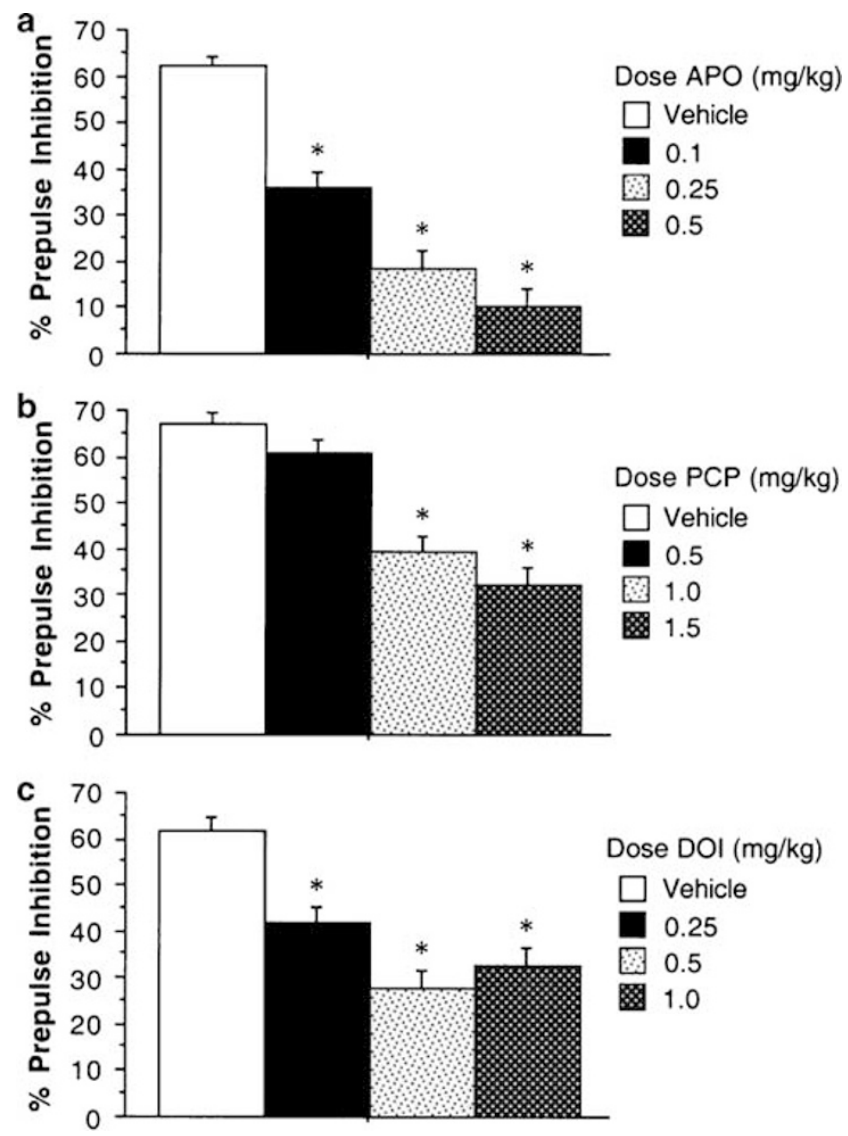

Figure I Percent PPI in dose-response studies with APO (a), PCP (b) and $\mathrm{DOI}(\mathrm{c})$. (*) $^{*} p<0.05$ vs vehicle dose.

Table I Statistics for Critical Comparisons

\begin{tabular}{|c|c|c|}
\hline Drug & Main effect of drug & Drug $\times$ trial type interaction \\
\hline \multicolumn{3}{|c|}{ (a) Drug effects on startle magnitude on PULSE and prepulse+pulse trials } \\
\hline APO & $F=2.77$, df $3,33, p<0.06$ & $F=6.85$, df $9,99, p<0.0001$ \\
\hline PCP & $F<1$ & $F=5.50$, df $9,81, p<0.0001$ \\
\hline DOI & $\mathrm{F}<1$ & $F=5.99$, df $9.99, p<0.0001$ \\
\hline \multicolumn{3}{|c|}{ (b) Drug effects on click-induced motor activity on S1 and S2 trials } \\
\hline APO & $F=9.85$, df $3,33, p<0.0001$ & $F=3.48$, df $3,33, p<0.03$ \\
\hline PCP & $F=3.31$, df $3,27, p<0.04$ & $F=4.22$, df $3,27, p<0.015$ \\
\hline DOI & $F=2.96$, df $3,33, p<0.05$ & $F=3.40$, df $3,33, p<0.03$ \\
\hline \multicolumn{3}{|c|}{ (c) Drug effects on startle magnitude on PULSE and prepulse+pulse trials } \\
\hline APO & $F=|I .7|, d f \mid, 23, p<0.003$ & $F=39.15$, df $3,69, p<0.0001$ \\
\hline PCP & $F=7.03, d f 1,21, p<0.015$ & $F=3.76$, df $3,63, p<0.02$ \\
\hline DOI & $\mathrm{F}<\mathrm{I}$ & $F=4.8 I$, df $3,60, p<0.005$ \\
\hline
\end{tabular}

significant interactions of dose $\times$ trial type (F for APO and $\mathrm{PCP}=6.85$ and 5.50, respectively; $p<0.0001$; Table 1a), and confirmed by appropriate posthoc comparisons. Interestingly, this pattern was not observed in DOI-treated rats: DOI suppressed startle magnitude on PULSE trials 

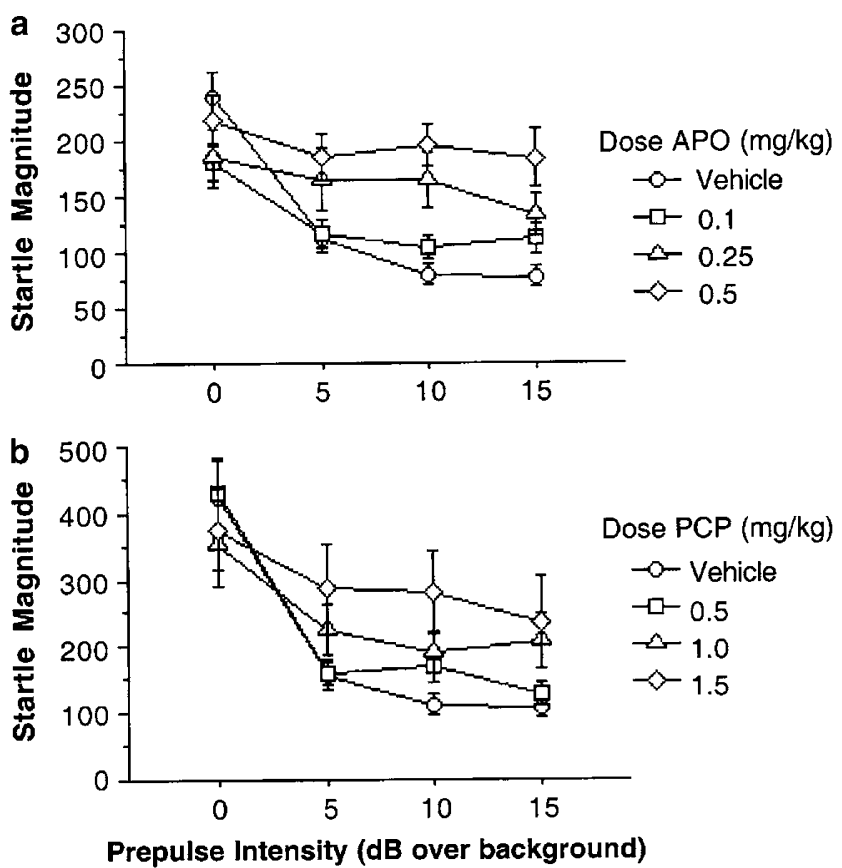

Figure 2 Raw startle magnitude on PULSE trials ( $0 \mathrm{~dB}$ prepulse) and trials in which PULSE was preceded by 5,10 , or $15 \mathrm{~dB}$ prepulses, in rats treated with APO (a) or PCP (b). Note that in both (a) and (b): (I) vehicletreated rats exhibit an orderly, intensity-dependent suppression of startle, and (2) rats treated with active drug doses exhibit a dose-dependent reduction in sensitivity to the startle-inhibiting effects of prepulses. This drug-induced reduction in the ability of prepulses to inhibit startle provides clear evidence for a loss of sensorimotor gating.

$(\mathrm{F}=3.84$, df $3,33, p<0.02)$, and did not significantly increase startle magnitude on prepulse + pulse trials (Figure 3a). However, because this was a within-subject dose-response study, it was possible to demonstrate increased startle magnitude on prepulse + pulse trials in the subset of rats for which DOI did not suppress startle on PULSE trials (Figure $3 \mathrm{~b}$ ). Thus, unlike the effects of APO and PCP, a selective loss of PPI after DOI was 'masked' by a reduction in startle magnitude, but could still be identified among rats less sensitive to the startle-suppressing effects of this drug.

A minimal motoric response to N40 clicks was detected in these studies, though the signal of the motoric response was less than $1 \%$ of the magnitude of responses elicited by startle stimuli, and only roughly 2-3 times as large as the 'background' motor response detected on NOSTIM trials (Figure 4). Nonetheless, this click-induced motor response exhibited several interesting properties. First, N40 amplitude was reduced in response to S2 vs S1 stimuli, with a typical S2/S1 ratio of about $0.38,0.28$, and 0.14 for APO, PCP, and DOI, respectively, that is, it exhibited 'gating' that paralleled the expected ERP response to such stimuli. Second, this S2/S1 ratio exhibited drug sensitivities that roughly mimicked those exhibited by PPI; ratios were increased (ie, gating was reduced) by APO, PCP, and DOI, and ANOVAs revealed significant drug $\times$ trial type ( $\mathrm{S} 1$ vs S2) interactions (Table $1 \mathrm{~b}$ ).

As a result of the low 'signal-to-noise' properties of this minimal motor response, compared to background
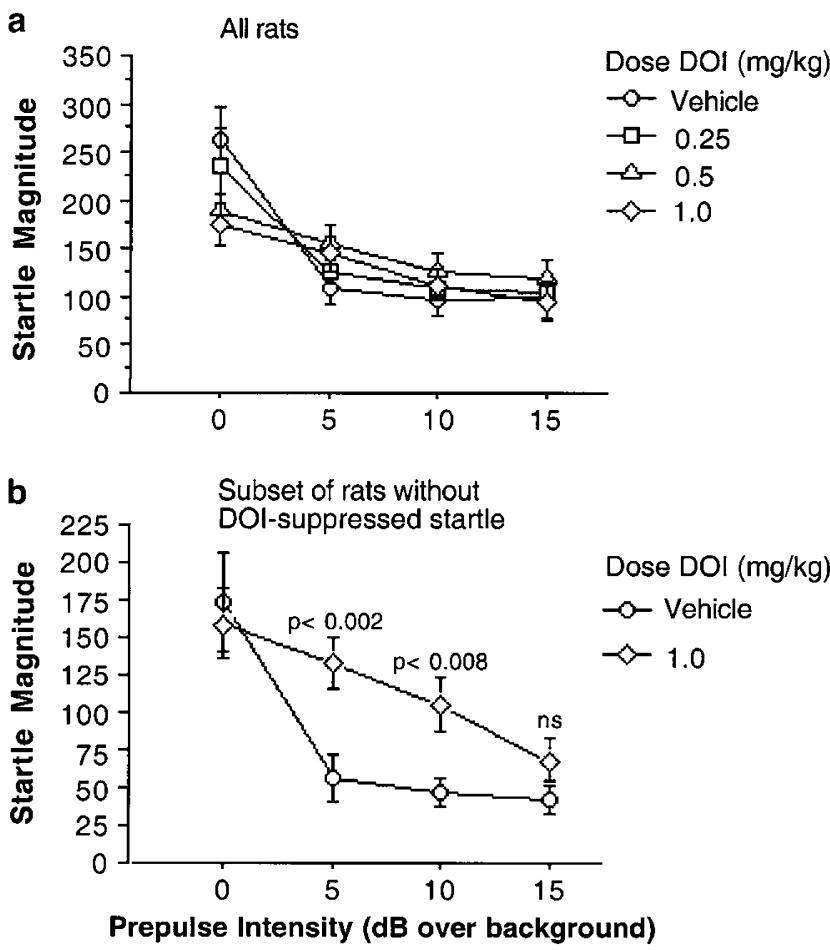

Figure 3 Raw startle magnitude on PULSE trials ( $0 \mathrm{~dB}$ prepulse) and trials in which PULSE was preceded by 5,10 , or $15 \mathrm{~dB}$ prepulses, in rats treated with DOI. (a) DOI produces a dose-dependent reduction in startle magnitude on PULSE trials that complicates a clear interpretation of drug effects on sensorimotor gating. (b) Startle magnitude from a subgroup of rats whose startle magnitude on PULSE trials was not significantly reduced by $\mathrm{DOI}(1.0 \mathrm{mg} / \mathrm{kg})$. Note that for this group, a DOl-induced loss of sensorimotor gating is evident via the reduced ability of prepulses to inhibit startle magnitude (dose $\times$ trial interaction: $F=6.05$, df 3, I5, $p<0.007$; posthoc comparisons: $p<0.002(5 \mathrm{~dB})$ and $p<0.008$ ( I $0 \mathrm{~dB})$ ). A comparable or slightly diminished separation was observed for the $0.5 \mathrm{mg} / \mathrm{kg}$ dose of DOI $(p<0.002(5 \mathrm{~dB})$ and $p<0.04(10 \mathrm{~dB}))$, but not for the $0.25 \mathrm{mg} / \mathrm{kg}$ dose (NS, all prepulse intensities).

NOSTIM activity, we examined the potential impact of drug-induced changes in NOSTIM activity on 'gating' of click-associated cage displacements. As we have reported previously, NOSTIM activity was increased in a dosedependent manner by each of the three drugs tested. Subtracting this motor 'background' from the motor signal generated by S1 and S2 revealed that increased S2/S1 ratios (reduced gating) for each drug primarily reflected a reduction in S1 magnitude (Figure 4). In other words, the apparent drug-induced disruption of paired-click motor 'gating' was actually a reflection of a drug-induced suppression of the motor response to $S 1$.

PPI did not correlate significantly with ratios of S2/S1induced motor responses. To enhance the likelihood of detecting such a correlation, S1- and S2-induced motor responses and PPI were examined on trials that were most temporally contiguous within the test session (first block of click pairs $v s$ first block of prepulse and PULSE stimuli). Examined for each drug and dose, for S2/S1 ratios either with or without correction for background NOSTIM activity, correlations of PPI and S2/S1 reached statistical significance for only one out of 24 possible pair-wise comparisons (data not shown). 
a

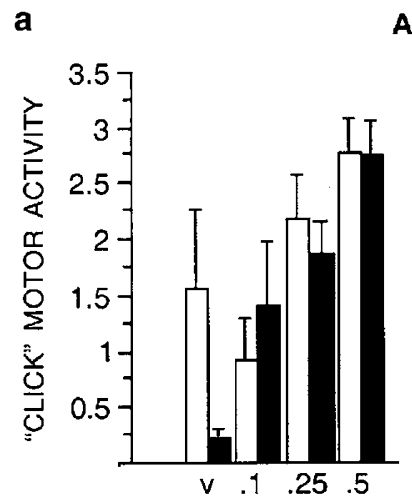

APO

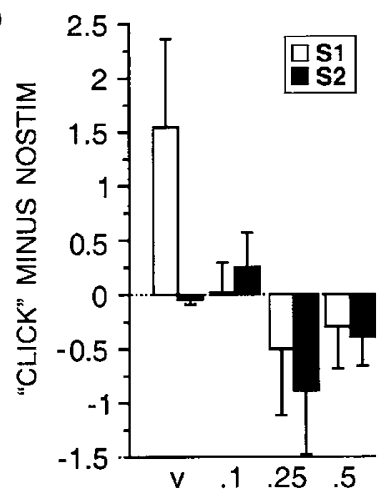

b<smiles>[B]CCC</smiles>
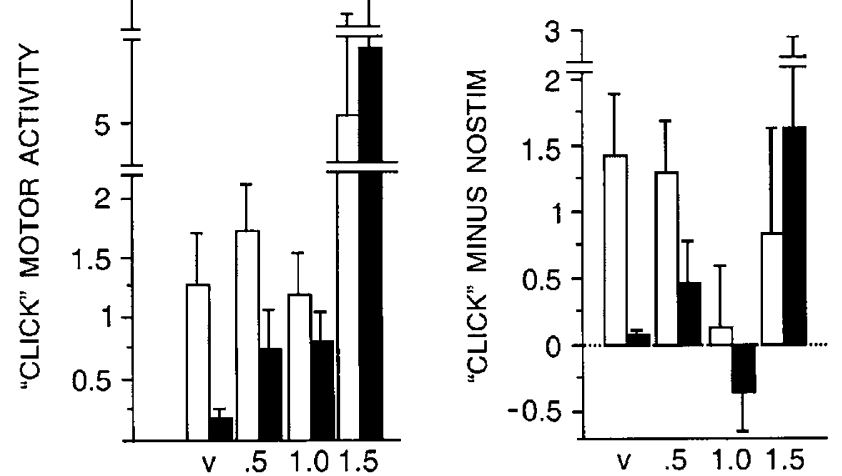

C

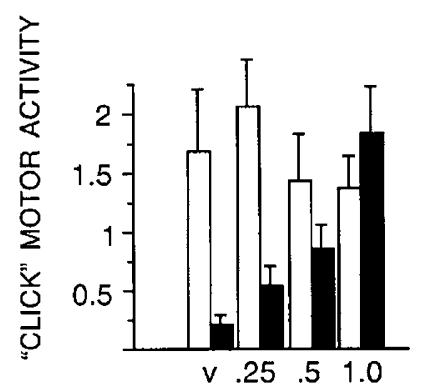

DOI

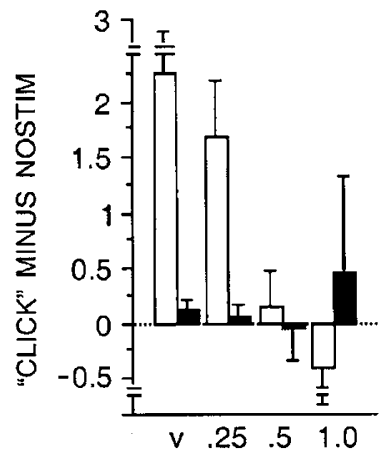

Figure 4 Motor activity on trials with SI and S2 'clicks'. Left side of figure shows 'click motor activity': peak response units 100 ms after SI or S2 'clicks' on same scale as startle magnitude in Figures 2-3. Note that motor activity on 'click' trials in vehicle-treated rats was: (I) $0.5-1.0 \%$ of the magnitude exhibited in response to PULSE trials; (2) greatly suppressed on S2 vs SI trials. Also note that S2 amplitude appeared to be enhanced preferentially in a dose-dependent manner by (a) APO, (b) PCP, and (c) $\mathrm{DO}$. Right side of figure shows the same data, minus response units exhibited on NOSTIM trials ('click minus nostim'). Note that, by removing NOSTIM background activity, S2 'gating' remains intact in vehicle-treated rats, and the most evident drug effect is a suppression of motor activity in response to SI clicks.

\section{Study 2. PPI/N40 Measures}

The effects of a single dose of APO $(0.5 \mathrm{mg} / \mathrm{kg})$, PCP $(1.5 \mathrm{mg} / \mathrm{kg})$, and DOI $(1.0 \mathrm{mg} / \mathrm{kg})$ on PPI and startle magnitude in these studies were consistent with the effects observed in the dose-response studies. As seen in Figure 5, PPI was reduced by APO $(\mathrm{F}=75.21, p<0.0001)$, PCP $(\mathrm{F}=11.38, p<0.005)$ and DOI $(\mathrm{F}=7.79, p<0.015)$, and
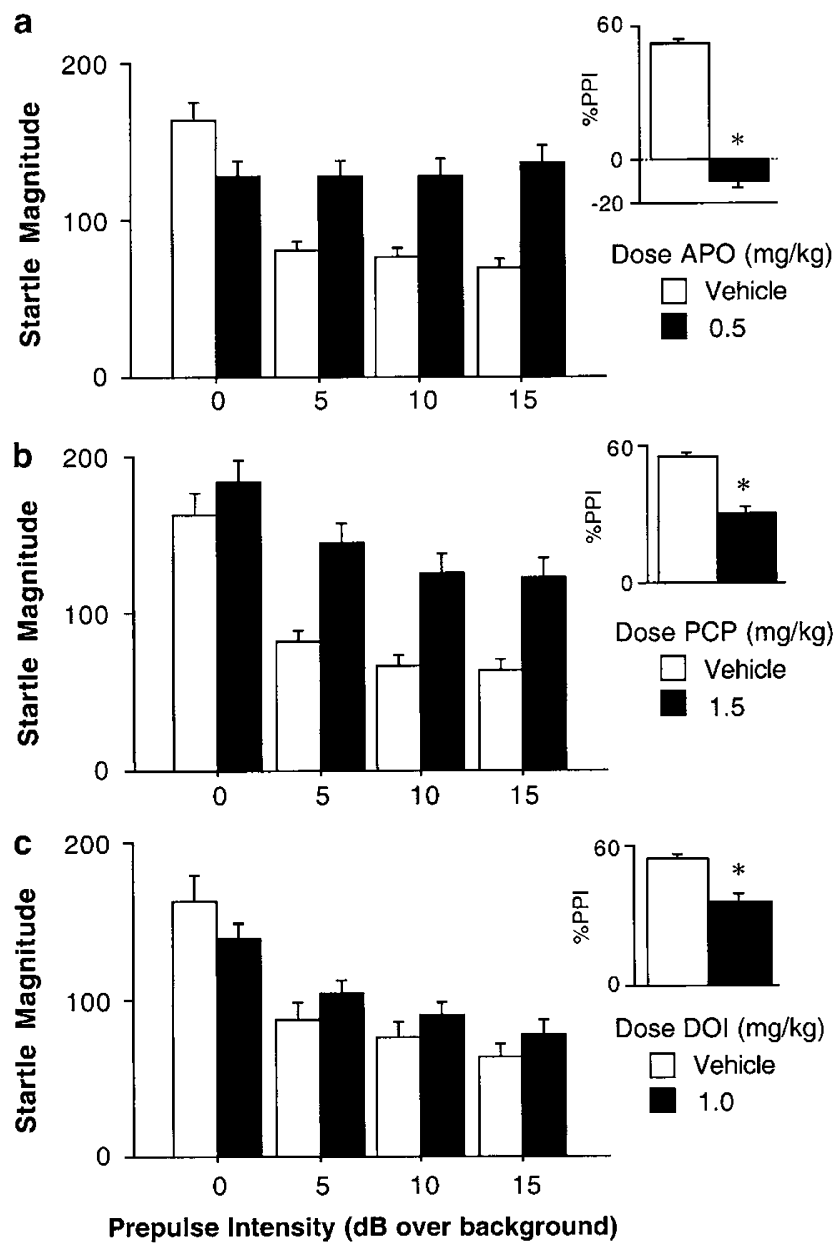

Figure 5 Raw startle magnitude on PULSE trials $(0 \mathrm{~dB}$ prepulse) and trials in which PULSE was preceded by 5,10 , or $15 \mathrm{~dB}$ prepulses, in rats treated with vehicle or a single dose of APO (a), PCP (b), or DOI (c). Note comparability to Figures 2-3. Insets reveal mean \%PPI, collapsed across prepulse intensities (* $p<0.05$ vs vehicle). APO study used a within-subject design, while PCP and DOI studies used between-subject designs in order to limit total number of tests for each rat.

these effects of APO and PCP (but not DOI) reflected selectively increased startle magnitude on prepulse + pulse trials. As with the dose-response studies, reduced PPI in DOI-treated rats was accompanied by a substantial reduction in startle magnitude on PULSE trials, in this case primarily in the initial PPI trial block (dose $\times$ block interaction: $\mathrm{F}=4.98, p<0.045$; block 3 effect of dose: $\mathrm{F}=5.97, p<0.03$ ) (Table 1c).

To assess test-retest stability of our measure of N40 amplitude in this combined PPI/N40 paradigm, we compared ERP amplitudes during two drug-free tests (no drug or vehicle injection), separated by 3-7 days. In some of these rats, an active dose test occurred between the two drug-free tests. Simple regression analyses revealed a highly significant test-retest correlation for drug-free $\mathrm{S} 1(r=0.69$, $n=15, p<0.005)$ and S2 $(r=0.72, n=15, p<0.003)$.

N40 magnitude on S1 and S2 trials for vehicle and active drug tests are seen in Figure 6, together with grand average waveforms for each drug condition and examples of typical individual rats from each dose group. $\mathrm{N} 40$ gating was clearly evident in the reduced $\mathrm{N} 40$ response to S2 compared 

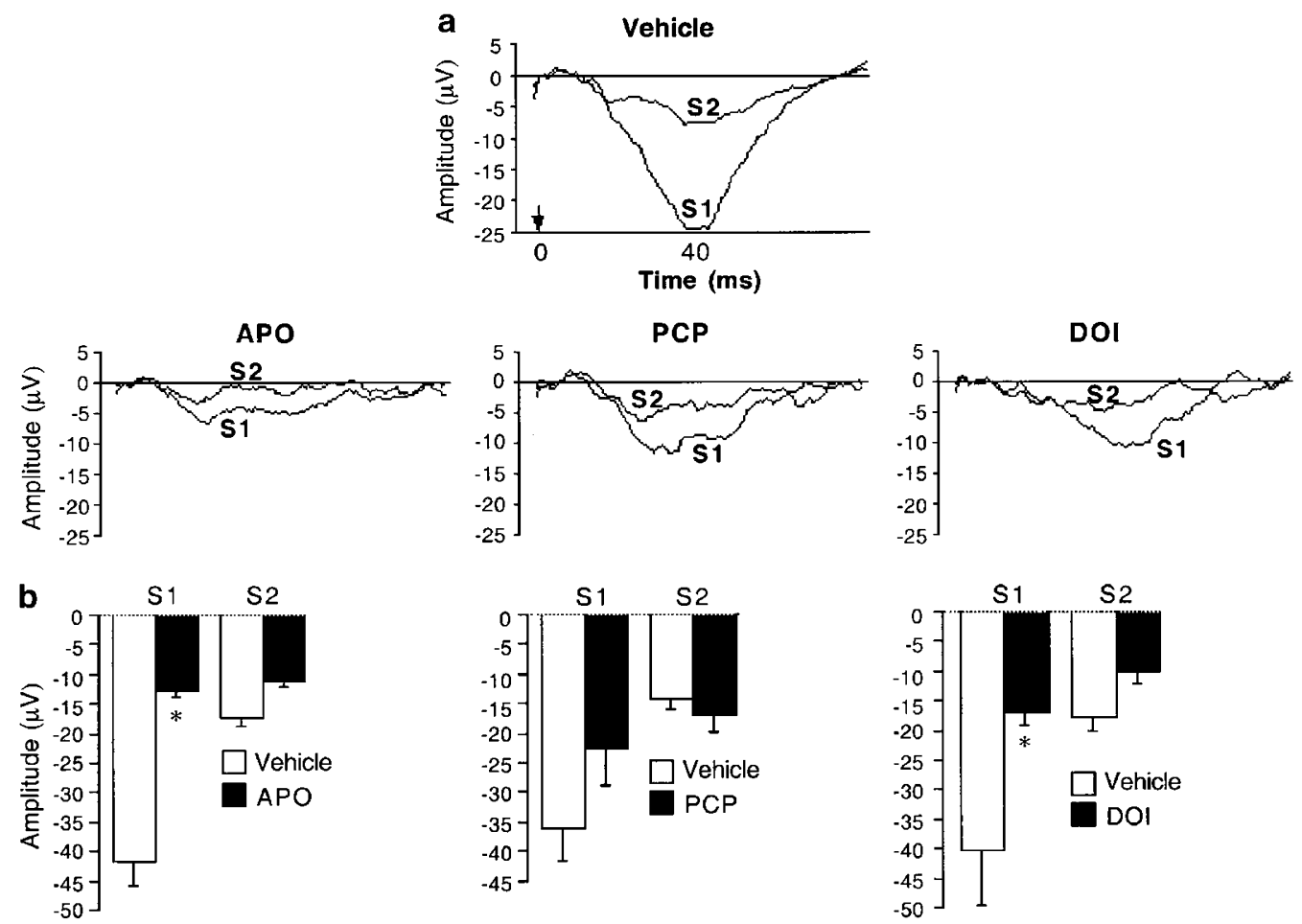

Figure 6 N40 ERP responses. (a) Grand average ERP waveforms in response to SI and S2, across all rats treated with vehicle (top), APO, PCP, or DOI. Note in vehicle treated rats, the peak negativity to SI at 40 ms poststimulus (stimulus at arrow), and reduced ('gated') amplitude to S2. This gating was disrupted by APO, PCP, and DOI, as a result of reduced SI amplitude. This was evident in grand average waveforms, and in peak N40 amplitudes, shown in b. (b) Peak N40 amplitudes in response to SI and S2 stimuli, in rats treated with vehicle or APO (left), PCP (middle), or DOI (right). Note significant reduction in $\mathrm{SI}$ amplitude after treatment with $\mathrm{APO}$ and $\mathrm{DOI}(* \mathrm{p}<0.05$ vs vehicle), and similar trend for this effect after treatment with $\mathrm{PCP}$.

to $\mathrm{S} 1$ for each individual vehicle-treated rat; the mean $\mathrm{S} 2 / \mathrm{S} 1$ ratios for vehicle-treated rats $(0.47,0.47$, and 0.46 in APO, PCP, and DOI studies, respectively) were consistent with those previously reported in the literature (Stevens et al, $1991,1995,1996,1998)$. Inspection of the data revealed that APO and DOI elevated the $S 2 / S 1$ ratio $(F=34.15$ and 5.11 ; $p^{\prime}<0.0001$ and $<0.04$, respectively) suggestive of a disruption of N40 gating; a similar trend was observed with PCP $(\mathrm{F}=4.03, p=0.06)$. APO, PCP and DOI suppressed ERP amplitude, with a disproportionate impact on S1 (main effects of dose: $\mathrm{F}=36.06$ and $5.42 ; p<0.0001$ and 0.035 , for APO and DOI, respectively; dose $\times$ ERP interactions: $\mathrm{F}=30.67,5.77$ and 4.39 and $4.91 ; p<0.001, p<0.03$ and $p=0.05$, for APO, PCP, and DOI, respectively). APO and DOI significantly reduced $\mathrm{S} 1$ amplitude $(\mathrm{F}=39.98$ and 5.93, $p<0.0001$ and 0.03 , respectively), but a trend for this effect with PCP did not reach significance $(\mathrm{F}=2.54, \mathrm{~ns})$. Thus, as with N40 click-induced motor activity, the apparent drug-induced disruption of N40 gating for each drug appeared to primarily reflect a suppression of the response to $\mathrm{S} 1$.

Simple regression analyses revealed no significant correlations between mean \%PPI (averaged across intensities) and S2/S1 ratios for vehicle or active doses in either APO, PCP, or DOI studies (data not shown). As a result of the within-subject design of the APO study, an 'APO effect' was calculated for each rat for both PPI (PPI vehicle minus PPI APO) and N40 (S2/S1 APO minus S2/S1 vehicle). Thus, a large positive number reflected a large APO effect for each measure. Interestingly, simple regression analysis revealed a significant negative correlation $(R=-0.67, n=18$, $p<0.003$ ), suggesting that a large disruption of PPI by APO predicted a small disruption of N40 gating in the same rats.

\section{DISCUSSION}

Findings from the dose-response studies demonstrate that it is feasible to detect orderly, parametrically sensitive PPI in rats in a session that also delivers click pairs that elicit N40 ERPs and N40 suppression. Furthermore, under these conditions, PPI exhibits three expected patterns of neurochemical sensitivity.

PPI in this mixed-stimulus paradigm was disrupted in a dose-dependent manner by APO, PCP, and DOI. While these effects of APO and PCP were attributable to a clear loss of sensorimotor gating. Those of DOI were more difficult to interpret due to a general suppression of startle magnitude, but could be identified clearly among a subgroup of rats less sensitive to the startle-suppressing effects of DOI.

'Paired-pulse inhibition' of motor activity in response to click pairs exhibits both similarities to, and differences from PPI. In this study, drug-induced changes in PPI could clearly be attributed to a loss in the motor-inhibitory effects of the prepulses. In contrast, drug-induced changes in the $\mathrm{S} 2 / \mathrm{S} 1$ motor response ratio reflect a disruption in the motor 
response to S1, rather than a clear loss of inhibitory gating effects per se. More generally, findings from the doseresponse study confirmed that the presence of click pairs in a test session do not interfere with PPI measures. Thus, we determined that it is both feasible and informative to measure PPI and N40 ERP gating contemporaneously in rats, to determine the neurochemical convergence and divergence of these putative measures of CNS-mediated gating processes. This line of investigation was pursued in the PPI/N40 ERP studies.

The PPI/N40 ERP studies demonstrated both strengths and limitations of this approach to contemporaneous assessments of these two gating measures. Both PPI and N40 ERP gating were detected easily; in particular, robust N40 ERPs and N40 suppression were identified that were consistent with numerous reports in the literature, in which N40 gating was assessed in sessions without startle stimuli. This capacity for contemporaneous acquisition made it possible to examine correlations of PPI and N40 gating within individual rats, at a time when the neurobiological state of the rat was essentially identical during assessment of both measures, and was thus not a potential source of variability. Contemporaneous measurements also allowed direct comparisons of drug effects on these two measures within the same rat, at a time when drug state could be assumed to be essentially identical for both measures (eg $v s$ rats tested at two different time points in two different sessions (Ellenbroek et al, 1999; de Bruin et al, 2001a,b)).

The main aim of these studies was to assess the neurochemical convergence and divergence of PPI and N40 ERP gating, based on the effects of drugs on these measures. In one parsimonious view of the findings, APO, PCP, and DOI were each found to disrupt both PPI and N40 ERP gating, demonstrating a neurochemical convergence of drug effects on these two measures. However, inspection of the data revealed that the loss of PPI after APO and PCP reflected a selective increase in responses to prepulse + pulse trials, that is, a loss of the inhibitory effectiveness of the prepulses. In contrast, the loss of N40 ERP gating after APO and PCP reflected a pronounced reduction in the magnitude of the ERP response to S1. In contrast to our expectations, compared to responses after vehicle treatment, there were no significant increases in the N40 responses to S2 after APO, PCP, or DOI. Unlike the effects observed with PPI, this apparent loss of N40 'gating' could alternatively be attributed to reduced sensory responsivity, an interpretation that would also be consistent with the observed patterns of $\mathrm{N} 40$ click-induced motor activity in the dose-response studies, described above. Thus, the simplest interpretation of the startle and ERP findings suggests a divergence in the neurobiological mechanisms responsible for drug-induced changes in PPI and N40 gating.

Our findings that dopamine and 5HT agonists reduce S1 response amplitude are consistent with previous reports. For example, Johnson et al (1998) reported that $2.5 \mathrm{mg} / \mathrm{kg}$ DOI reduced S1 response amplitude by $36 \%$ (p. 648, Table $2)$. Other groups have reported elevated $S 1$ response amplitude in rodents after administration of antipsychotic agents with potent 5HT2 antagonism, such as olanzapine (Maxwell et al, 2004). However, Johnson et al (1998) also reported that DOI caused a proportionately larger reduction in S2 response magnitude (57\%), resulting in a reduction in the S2/S1 ratio. The fact that we did not observe a proportionately larger reduction in S2 amplitude in the present study may reflect the fact that we were limited to a much lower dose of DOI in the present study $(1.0 \mathrm{mg} / \mathrm{kg} v \mathrm{~s}$ $2.5 \mathrm{mg} / \mathrm{kg}$ in Johnson et al (1998)), due to the potent startlesuppressant effects of this drug. Importantly, with this lower dose of DOI, we were able to detect a significant disruption in sensorimotor gating (PPI) among rats less sensitive to its startle-suppressant effects.

What are the implications of the convergent and divergent aspects of PPI and ERP gating, as detected in these studies? One might speculate that, to the degree that there is overlap in the neurochemical sensitivity of these measures, the relative loss of both PPI and ERP gating in some patients with schizophrenia might reflect a common disturbance, or pattern of disturbances, in brain chemistry. A weakness in this interpretation is the lack of evidence for convergence of PPI and P50 gating deficits within the same schizophrenia patients. In fact, evidence appears to be emerging for a divergence of these deficits within the same schizophrenia-spectrum patients (Cadenhead et al, 2002), that is, individual schizophrenia and schizotypal patients may most likely have deficits in one but not both of these measures.

Perhaps even more complex is the understanding of the likely mechanistic divergence of these measures. Thus, reduced 'gating' in PPI reflects a loss of the inhibitory effects of the prepulse, while reduced 'gating' of the N40 ERP reflects a reduction in the magnitude of the ERP response to the $\mathrm{S} 1$. One might speculate that a common process - reduced stimulus responsivity - might contribute to drug-induced decreases of both prepulse effectiveness in PPI, and of S1 ERP amplitude. The possibility that druginduced reductions in responsivity contribute to reduced PPI is not easily reconciled with the fact that PPI is not consistently related to startle magnitude. Startle can be reduced, elevated, or unchanged at a time when drugs have completely eliminated PPI (cf. Swerdlow et al, 2000). Furthermore, reduced PPI after APO or PCP is actually associated with increased startle magnitude on prepulse + pulse trials, arguing against a generalized reduction in stimulus responsivity. Conceivably, drug effects on PPI might reflect a selective reduction in responsivity to weak stimuli (eg prepulses), but this hypothesis would not be consistent with evidence that drugs that reduce or eliminate PPI in response to relatively more intense prepulses (eg 10-15 dB over background) can actually increase PPI in response to weak $(1-2 \mathrm{~dB})$ prepulses (eg Swerdlow et al, 2001b).

The present studies clearly do not address all of the potential sources of neurochemical convergence and divergence of PPI and N40 suppression. Studies in progress are focusing on the impact of D1 blockade on concurrent measurements of PPI and ERP suppression, the ability of antipsychotic agents to oppose the PPI- and N40-disruptive effects of dopamine agonists and NMDA antagonists, and the effects of selective intracerebral manipulations of limbic and striatal circuitry on these measures. Other studies are comparing the impact of specific developmental manipulation on PPI and ERP suppression.

The link between reduced ERP suppression and diminished responsivity to $S 1$ stimuli has been reported 
previously in both preclinical (Adler et al, 1986; de Bruin et al, 1999, 2001a,b; Stevens et al, 1991, 1996) and clinical studies (Adler et al, 1982; Boutros et al, 1991; Freedman et al, 1983; Nagamoto et al, 1989; Myles-Worsley 2002), but the basis for this association remains a matter of some contention in need of databased resolution. In these clinical studies, reduced S1 magnitude often contributes to an increase in S2/S1 ratio in schizophrenia groups, even to a greater degree than does an absolute increase in S2 magnitude. This observation may not detract from the importance of such deficits: conceivably, diminished S1 ERP magnitude in schizophrenia, and in preclinical models, might reflect genetic and neural events of direct relevance to the pathophysiology of this disorder. However, the present findings support existing evidence that impaired 'gating' of a second stimulus per se need not be invoked as an explanation for such phenomena, but rather, that such deficits are fully detected in the diminished response to a single stimulus presentation. In this regard, there is a clear divergence between deficits in PPI and ERP suppression, and this divergence was detected in the present study, via their contemporaneous measurement in the same animals.

\section{ACKNOWLEDGEMENTS}

This work was supported by MH01436 and MH53484 (NRS) and MH42228 (DLB, NRS, MAG, KES). MAG holds an equity interest in San Diego Instruments.

\section{REFERENCES}

Adams CE, Stevens KE (1998). Inhibition of nitric oxide synthase disrupts inhibitory gating of auditory responses in rat hippocampus. J Pharmacol Exp Ther 287: 760-765.

Adler LE, Pachtman E, Franks RD, Pecevich M, Waldo MC, Freedman R (1982). Neurophysiological evidence for a defect in neuronal mechanisms involved in sensory gating in schizophrenia. Biol Psychiatry 17: 639-654.

Adler LE, Rose G, Freedman R (1986). Neurophysiological studies of sensory gating in rats: effects of amphetamine, phencyclidine, and haloperidol. Biol Psychiatry 21: 787-798.

Bolino F, DiMichele V, DiCicco L, Manna V, Daneluzzo E, Casacchia M (1994). Sensorimotor gating and habituation evoked by electro-cutaneous stimulation in schizophrenia. Biol Psychiatry 36: 670-679.

Boutros NN, Korzyukov O, Jansen B, Feingold A, Bell M (2004). Sensory gating deficits during the mid-latency phase of information processing in medicated schizophrenia patients. Psychiatry Res 126: 203-215.

Boutros NN, Uretsky N, Berntson G, Bornstein R (1994). Effects of cocaine on sensory inhibition in rats: preliminary data. Biol Psychiatry 36: 242-248.

Boutros NN, Zouridakis G, Overall J (1991). Replication and extension of P50 findings in schizophrenia. Clin Electroencephalogr 22: 40-45.

Braff D, Stone C, Callaway E, Geyer M, Glick I, Bali L (1978). Prestimulus effects on human startle reflex in normals and schizophrenics. Psychophysiology 14: 339-343.

Braff DL, Freedman R (2002). The importance of endophenotypes in studies of the genetics of schizophrenia. Neuropsychopharmacology: The Fifth Generation of Progress. Lippincott Williams \& Wilkins: Baltimore, MD. pp 703-716.

Braff DL, Geyer MA, Light GA, Sprock J, Perry W, Cadenhead KC et al (2001a). Impact of prepulse characteristics on the detection of sensorimotor gating deficits in schizophrenia. Schizophr Res 49: $171-178$.

Braff DL, Geyer MA, Swerdlow NR (2001b). Human studies of prepulse inhibition of startle: normal subjects, patient groups, and pharmacological studies. Psychopharmacology 156: 234-258.

Braff DL, Swerdlow NR, Geyer MA (1999). Symptom correlates of prepulse inhibition deficits in male schizophrenia patients. $\mathrm{Am} J$ Psychiatry 156: 596-602.

Brenner CA, Edwards CR, Carroll CA, Kieffaber PD, Hetrick WP (2004). P50 and acoustic startle gating are not related in healthy participants. Psychophysiology 41: 702-708.

Cadenhead KS, Light GA, Geyer MA, Braff DL (2000a). Sensory gating deficits assessed by the P50 event-related-potential in subjects with schizotypal personality disorder. Am J Psychiatr 157: 55-59.

Cadenhead KS, Light GA, Geyer MA, McDowell JE, Braff DL (2002). Neurobiological measures of schizotypal personality disorder: defining an inhibitory endophenotype? Am J Psychiatr 159: 869-871.

Cadenhead KS, Swerdlow NR, Shafer KM, Diaz M, Braff DL (2000b). Modulation of the startle response and startle laterality in relatives of schizophrenia patients and schizotypal personality disordered subjects: evidence of inhibitory deficits. $\mathrm{Am} J$ Psychiatr 157: 1660-1668.

Clementz BA, Geyer MA, Braff DL (1998). Poor P50 suppression among schizophrenia patients and their first-degree biological relatives. Am J Psychiatr 155: 1691-1694.

de Bruin NM, Ellenbroek BA, Cools AR, Coenen AM, van Luijtelaar EL (1999). Differential effects of ketamine on gating of auditory evoked potentials and prepulse inhibition in rats. Psychopharmacology 142: 9-17.

de Bruin NM, Ellenbroek BA, van Luijtelaar EL, Cools AR, Stevens KE (2001a). Hippocampal and cortical sensory gating in rats: effects of quinpirole microinjections in nucleus accumbens core and shell. Neuroscience 105: 169-180.

de Bruin NM, van Luijtelaar EL, Cools AR, Ellenbroek BA (2001b). Auditory information processing in rat genotypes with different dopaminergic properties. Psychopharmacology 156: 352-359.

Ellenbroek BA, van Luijtelaar G, Frenken M, Cools AR (1999). Sensory gating in rats: lack of correlation between auditory evoked potential gating and prepulse inhibition. Schizophr Bull 25: 777-788.

Erwin RJ, Turetsky BI, Moberg P, Gur RC, Gur RE (1998). P50 abnormalities in schizophrenia: relationship to clinical and neuropsychological indices of attention. Schizophr Res 33: 157-167.

Freedman R, Adler LE, Gerhardt GA, Waldo M, Baker N, Rose GM et al (1987). Neurobiological studies of sensory gating in schizophrenia. Schizophr Bull 13: 669-678.

Freedman R, Adler LE, Waldo MC, Pachtman E, Franks RD (1983). Neurophysiological evidence for a defect in inhibitory pathways in schizophrenia: comparison of medicated and drug-free patients. Biol Psychiatry 18: 537-551.

Freedman R, Coon H, Myles-Worsley M, Orr-Urtreger A, Olincy A, Davis A et al (1997). Linkage of a neurophysiological deficit in schizophrenia to a chromosome 15 locus. Proc Natl Acad Sci USA 94: 587-592.

Geyer MA, Krebs-Thomson K, Braff DL, Swerdlow NR (2001). Pharmacological studies of prepulse inhibition models of sensorimotor gating deficits in schizophrenia: a decade in review. Psychopharmacology 156: 117-154.

Geyer MA, Swerdlow NR (1998). The startle reflex and its use in preclinical measures of sensorimotor gating. In: Crawley $\mathrm{JN}$, Skolnick P (eds). Current Protocols in Neuroscience. John Wiley \& Sons: New York, 8.7.1-8.7.15.

Graham F (1975). The more or less startling effects of weak prestimuli. Psychophysiology 12: 238-248. 
Grunwald T, Boutros NN, Pezer N, von Oertzen J, Fernandez G, Schaller $\mathrm{C}$ et al (2003). Neuronal substrates of sensory gating within the human brain. Biol Psychiatr 53: 511-519.

Johnson RG, Stevens KE, Rose GM (1998). 5-Hydroxytryptamine2 receptors modulate auditory filtering in the rat. J Pharmacol Exp Ther 285: 643-650.

Judd LL, McAdams L, Budnick B, Braff DL (1992). Sensory gating deficits in schizophrenia: new results. Am J Psychiatr 149: 488-493.

Kumari V, Soni W, Sharma T (1999). Normalization of information processing deficits in schizophrenia with clozapine. $\mathrm{Am} \mathrm{J}$ Psychiatr 156: 1046-1051.

Light GA, Braff DL (2001). Measuring P50 suppression and prepulse inhibition in a single recording session. Am J Psychiatr 158: 2066-2068.

Light GA, Braff DL (2005). Mismatch negativity deficits are associated with poor functioning in schizophrenia patients. Arch Gen Psychiatr 62: 127-136.

Ludewig K, Geyer MA, Etzensberger M, Vollenweider FX (2002). Stability of the acoustic startle reflex, prepulse inhibition, and habituation in schizophrenia. Schizophr Res 55: 129-137.

Mansbach RS, Geyer MA (1989). Effects of phencyclidine and phencyclidine biologs on sensorimotor gating in the rat. Neuropsychopharmacology 2: 299-308.

Maxwell CR, Liang Y, Weightman BD, Kanes SJ, Abel T, Gur RE et al (2004). Effects of chronic olanzapine and haloperidol differ on the mouse N1 auditory evoked potential. Neuropsychopharmacology 29: 739-746.

Miller CL, Bickford PC, Luntz-Leybman V, Adler LE, Gerhardt GA, Freedman R (1992). Phencyclidine and auditory sensory gating in the hippocampus of the rat. Neuropharmacology 31: 1041-1048.

Miyazato H, Skinner RD, Garcia-Rill E (1999). Sensory gating of the P13 midlatency auditory evoked potential and the startle response in the rat. Brain Res 822: 60-71.

Myles-Worsley M (2002). P50 sensory gating in multiplex schizophrenia families from a Pacific island isolate. $\mathrm{Am} \mathrm{J}$ Psychiatry 159: 2007-2012.

Nagamoto HT, Adler LE, Waldo MC, Freedman R (1989). Sensory gating in schizophrenics and normal controls: effects of changing stimulation interval. Biol Psychiatry 25: 549-561.

Oranje B, van Berckel BN, Kemmer C, van Ree JM, Kahn RS, Verbaten MN (1999). P50 suppression and prepulse inhibition of the startle reflex in humans: a correlational study. Biol Psychiatry 45: 883-890.

Oranje B, van Oel CJ, Gispen-De Wied CC, Verbaten MN, Kahn RS (2002). Effects of typical and atypical antipsychotics on the prepulse inhibition of the startle reflex in patients with schizophrenia. J Clin Psychopharmacol 22: 359-365.

Parwani A, Duncan EJ, Bartlett E, Madonick SH, Efferen TR, Rajan $\mathrm{R}$ et al (2000). Impaired prepulse inhibition of acoustic startle in schizophrenia. Biol Psychiatry 47: 662-669.
Schwarzkopf SB, Lamberti JS, Smith DA (1993). Concurrent assessment of acoustic startle and auditory P50 evoked potential measures of sensory inhibition. Biol Psychiatry 33: 815-828.

Shepard PD, Joy B, Clerkin L, Schwarcz R. (2003). Micromolar brain levels of kynurenic acid are associated with a disruption of auditory sensory gating in the rat. Neuropsychopharmacology 28: 1454-1462.

Siegel C, Waldo M, Mizner G, Adler LE, Freedman R (1984). Deficits in sensory gating in schizophrenic patients and their relatives. Evidence obtained with auditory evoked responses. Arch Gen Psychiatr 41: 607-612.

Sipes TE, Geyer MA (1994). Multiple serotonin receptor subtypes modulate prepulse inhibition of the startle response in rats. Neuropharmacology 33: 441-448.

Stevens KE, Fuller LL, Rose GM (1991). Dopaminergic and noradrenergic modulation of amphetamine-induced changes in auditory gating. Brain Res 555: 91-98.

Stevens KE, Luthman J, Lindqvist E, Johnson RG, Rose GM (1996). Effects of neonatal dopamine depletion on sensory inhibition in the rat. Pharmacol Biochem Behav 53: 817-823.

Stevens KE, Meltzer J, Rose GM (1995). Nicotinic cholinergic normalization of amphetamine-induced loss of auditory gating in freely moving rats. Psychopharmacology 119: 163-170.

Stevens KE, Nagamoto H, Johnson RG, Adams CE, Rose GM (1998). Kainic acid lesions in adult rats as a model of schizophrenia: changes in auditory information processing. Neuroscience 82: 701-708.

Swerdlow NR, Bongiovanni MJ, Tochen LS, Shoemaker JM (2005). Separable noradrenergic and dopaminergic regulation of prepulse inhibition in rats: Implications for predictive validity and Tourette Syndrome. Abstr Soc Biol Psychiatry 57: 39S.

Swerdlow NR, Braff DL, Geyer MA (2000). Animal models of deficient sensorimotor gating: What we know, what we think we know, and what we hope to know soon. Behav Pharmacol 11: 185-204.

Swerdlow NR, Geyer M, Braff D, Koob GF (1986). Central dopamine hyperactivity in rats mimics abnormal acoustic startle in schizophrenics. Biol Psychiatry 21: 23-33.

Swerdlow NR, Geyer MA, Braff DL (2001a). Neural circuitry of prepulse inhibition of startle in the rat: current knowledge and future challenges. Psychopharmacology 156: 194-215.

Swerdlow NR, Platten A, Shoemaker J, Pitcher L, Auerbach P (2001b). Effects of pergolide on sensorimotor gating of the startle reflex in rats. Psychopharmacology 158: 230-240.

Waldo MC, Adler LE, Freedman R (1988). Defects in auditory sensory gating and their apparent compensation in relatives of schizophrenics. Schiz Res 1: 19-24.

Wan FJ, Taaid N, Swerdlow NR (1996). Do D1/D2 interactions regulate prepulse inhibition in rats? Neuropsychopharmacology 14: $265-274$

Weike AI, Bauer U, Hamm AO (2000). Effective neuroleptic medication removes prepulse inhibition deficits in schizophrenia patients. Biol Psychiatry 47: 61-70. 